\title{
Tendência dos índices de instabilidade na américa do sul
}

\author{
Trend of the instability indices in south america
}

\author{
Rute Costa Ferreira ${ }^{1}$, Michelle S. Reboita ${ }^{2}$, Dirceu L. Herdies ${ }^{3}$ e Cesar A. A. Beneti ${ }^{4}$ \\ ${ }^{1}$ Meteorologista, Centro de Previsão de Tempo e Estudos Climáticos, Instituto Nacional de Pesquisas Espaciais. Cachoeira \\ Paulista, SP, Brasil \\ ${ }^{2}$ Dra., Instituto de Recursos Naturais, Universidade Federal de Itajubá. Itajubá, MG, Brasil. \\ ${ }^{3}$ Dr.,Centro de Previsão de Tempo e Estudos Climáticos, Instituto Nacional de Pesquisas Espaciais, SP, Brasil \\ ${ }^{4}$ Dr., Sistema Meteorológico do Paraná. Curitiba, PR, Brasil
}

\begin{abstract}
Resumo
Os indices de instabilidade revelam a estrutura vertical da atmosfera com base na temperatura do ar e umidade a fim de identificar instabilidade ou estabilidade. Em caso de instabilidade, estes fornecem um indicativo da intensidade dos sistemas precipitantes. O indice K indica o potencial para tempestades com base na taxa vertical de variação da temperatura, na umidade na baixa troposfera e na extensão da camada úmida. O indice Total Totals (TT) combina temperatura e umidade em baixos níveis com a temperatura em níveis médios. Devido à grande importância destes indices na previsão de tempo e a falta de estudos quanto à tendência destes parâmetros na América do Sul, este trabalho teve como objetivo determinar os índices $\mathrm{K}$ e TT para os horários sinóticos, realizar uma climatologia e calcular a tendência sazonal destes indices. Os resultados mostraram que no interior do continente, maiores valores dos índices $K$ e TT são obtidos no verão, devido principalmente à variação sazonal da temperatura, além da precipitação associada ao regime monçônico especialmente no sudeste do Brasil. Há tendência de aumento nos valores de ambos os índices no norte da Amazônia e tendência de decréscimo a noroeste da Argentina e sudoeste da Bolívia.
\end{abstract}

Palavras-chave: Climatologia. Índice K. Índice TT.

\begin{abstract}
The instability indices evaluate the vertical structure of the atmosphere related to temperature and air humidity aiming to identify instability or stability. Where exists instability, these provide an indication of the intensity of precipitation systems. The $\mathrm{K}$ index indicates the potential for storms based on the lapse rate and humidity in the lower troposphere and in the extension of the wet layer. The Total Totals (TT) index combines temperature and moisture at low levels with the temperature on middle levels. Due to the importance of these indices in weather forecast and the absence of studies of the tendency of these parameters in South America, this research aimed to determine the K and TT indices for the synoptic hours make a climatology and calculate the seasonal trend of these indices. Results showed that on the continent higher values of the indices $K$ and TT are obtained in the summer, mainly due to seasonal variation of temperature, in addition to rainfall associated with the monsoon particularly in southeastern Brazil. There is an increasing trend in the values of both indices in the northern Amazon and decreasing trend in the northwest of Argentina and southwest Bolivia
\end{abstract}

Keywords: Climatology, K index, TT index. 


\section{Introdução}

A atmosfera pode ser classificada de acordo com seu estado de equilíbrio termodinâmico. De maneira simplificada, ela é considerada estável quando não há movimentos turbulentos expressivos na vertical, caracterizada geralmente por fracos ventos. No caso de estabilidade atmosférica, as camadas de ar mais próximas da superfície são mais frias do que as camadas logo acima destas, gerando uma camada de estabilidade. Caso uma parcela de ar seja deslocada verticalmente nestas condições, ela tende a retornar a sua posição inicial. A atmosfera neutra é caracterizada por condições de ventos moderados e caso exista um deslocamento vertical de uma parcela de ar, esta tende a permanecer onde foi deslocada. Já a atmosfera é considerada instável na ocorrência de camadas de ar consideravelmente mais quentes próximas da superfície e mais frias logo acima. Esse perfil de intenso gradiente de temperatura com o aumento da altura é comum na primavera e verão devido ao grande aquecimento diurno da superfície provocado pela maior incidência de radiação solar nesta estação do ano em grande parte do continente. Esse aspecto favorece a convecção e, consequentemente, a instabilização da atmosfera, favorecendo a formação de nuvens, precipitação e principalmente a ocorrência de tempestades convectivas.

Para avaliar se a atmosfera é estável ou instável são utilizados índices de instabilidade. De acordo com Beneti e Silva Dias (1986), os índices de instabilidade podem ser definidos como parâmetros numéricos de cálculo objetivo que visam estabelecer uma relação entre a ocorrência de tempestades e a circulação de meso ou grande escala nas quais estão imersas, e para tentar prognosticá-las, utilizam-se perfis verticais de vento, temperatura e umidade. Segundo Escobar e Andrade (2010), em caso de instabilidade atmosférica, os índices sugerem qual a intensidade das chuvas e se há possibilidade de queda de granizo, sendo úteis na previsão de eventos extremos.

Segundo Fragoso (1998), os diversos índices de instabilidade são diferenciados por tipos de parâmetros, em função da sua complexidade e dos indicativos que fornecem. Os índices K e TT são índices que se baseiam no princípio de que a instabilidade pode ser inferida apenas com base nas diferenças de condições de temperatura e umidade entre dois ou três níveis isobáricos. Com base nestes dados é possível estimar a estrutura vertical da atmosfera analisando os principais processos atmosféricos que influenciam a formação de convecção e futura dissipação. Esses índices são muito úteis e, por serem simples de calcular, quando utilizados em conjunto com a meteorologia sinótica, eles ajudam tanto em pesquisas quanto na parte de previsão do tempo. Já de acordo com Nascimento (2005), os índices são capazes de apontar condições potencialmente favoráveis à ocorrência de tempestades, porém não exploram a questão da iniciação convectiva, e há limitações em usar os valores limítrofes para o hemisfério sul, uma vez que estes limiares foram desenvolvidos para o hemisfério norte. Na América do Sul, os limiares dos índices possuem melhor desempenho na região Sul do Brasil e em latitudes médias.

Os diversos índices de instabilidade podem ser combinados e usados na previsão do tempo operacional. Pinheiro et al. (2013) mostram uma ferramenta objetiva usada para previsão de tempo severo, na qual gera cartas que apontam regiões propensas a nebulosidade, pancadas de chuvas com trovoadas, tempestades, e ocorrência de granizo, implementada na rotina operacional do CPTEC/INPE. Estas cartas são importantes também para a caracterização do tipo de precipitação, permitindo através dos limares usados estabelecer diferentes padrões convectivos. A análise do índice TT é conveniente para prever eventos severos associados à queda de granizo. Se ambos valores de K e TT são altos, a tendência é ter chuvas torrenciais. Se o valor de K é baixo e TT é alto, então a tendência é ter tempestades severas (Escobar e Andrade, 2010). Devido à relevância destes índices e da falta de estudos dos mesmos na América do Sul, este trabalho teve como objetivo realizar uma climatologia sazonal dos índices K e TT a fim de avaliar a tendência deles no período de estudo.

\section{Metodologia}

Com o intuito de realizar uma climatologia dos índices de instabilidade $\mathrm{K}$ e TT, foram utilizados dados de temperatura e umidade do ar obtidos da reanálise ERA-Interim do ECMWF (European Centre for Medium -Range Weather Forecasts) com resolução horizontal de $0.75^{\circ}$ (disponíveis em: apps.ecmwf.int/datasets/data/ interim-full-daily), para os quatro horários sinóticos (00, 06, 12 e 18 UTC) no período de janeiro de 1979 e dezembro 2012.

A área em estudo é compreendida entre as latitudes $60^{\circ} \mathrm{S}$ a $15^{\circ} \mathrm{N}$ e longitude de $270^{\circ}$ a $330^{\circ} \mathrm{W}$, e os dados foram obtidos em três níveis de pressão da atmosfera $(850,700$ e $500 \mathrm{hPa})$. Com a umidade relativa (Ur) e temperatura $\left(\mathrm{T}, \mathrm{em}{ }^{\circ} \mathrm{C}\right)$ foi calculada a temperatura de ponto de orvalho (Td) nos níveis de 700 e $850 \mathrm{hPa}$ a partir das equações (VIANELLO E ALVES, 1991), para T $>0^{\circ} \mathrm{C}$ :

$$
\mathrm{T}_{\mathrm{d}}=\frac{237,3 *\left(\log _{10} \mathrm{Ur}+\frac{7,5 * \mathrm{~T}}{237,3+\mathrm{T}}\right)}{7,5-\log _{10} \mathrm{Ur}-\frac{7,5 * \mathrm{~T}}{237,3+\mathrm{T}}}
$$

E para $\mathrm{T}<0^{\circ} \mathrm{C}$ :

$$
\mathrm{T}_{\mathrm{d}}=\frac{243,33 *\left(\log _{10} \mathrm{Ur}+\frac{7,665^{*} \mathrm{~T}}{243,33+\mathrm{T}}\right)}{7,665-\log _{10} \mathrm{Ur}-\frac{7,665 * \mathrm{~T}}{243,33+\mathrm{T}}}
$$


Foi desenvolvido um algoritmo no software Matlab para realizar os cálculos numéricos. Após o cálculo da temperatura de ponto de orvalho, os índices Ke TT foram calculados para cada horário sinótico bem como a climatologia sazonal e anual de cada índice, e realizada uma análise de tendência.

\subsection{Cálculo dos índices K e TT}

$\mathrm{O}$ índice $\mathrm{K}$ indica o potencial para tempestades com base na taxa vertical de variação da temperatura, na umidade na baixa troposfera e na extensão da camada úmida. Este índice pode ser calculado através da equação (George, 1960):

$$
\mathrm{K}=\left(\mathrm{T}_{850}-\mathrm{T}_{500}\right)+\mathrm{T}_{\mathrm{d} 850}-\left(\mathrm{T}_{700}-\mathrm{T}_{\mathrm{d} 700}\right)
$$

A estimativa da taxa vertical de variação da temperatura é calculada pela diferença entre as temperaturas de $850\left(\mathrm{~T}_{850}\right)$ e $500 \mathrm{hPa}\left(\mathrm{T}_{500}\right)$, a umidade na baixa troposfera é dada pela temperatura do ponto de orvalho de $850 \mathrm{hPa}$ $\left(\mathrm{T}_{\mathrm{d} 850}\right)$, e a extensão vertical da camada úmida é calculada pela diferença entre a temperatura do ar $\left(\mathrm{T}_{700}\right)$ e do ponto de orvalho $\left(\mathrm{T}_{\mathrm{d} 700}\right)$, ambas em $700 \mathrm{hPa}$.

$\mathrm{O}$ índice TT combina a temperatura de níveis médios e a umidade em baixos níveis. O TT é usado para avaliar o entranhamento de ar frio na troposfera média, sendo relevante para previsão de eventos severos associados à queda de granizo. Este índice é obtido através da soma dos índices Cross Totals (CV) e Vertical Totals (VT) (MILLER, 1972):

$$
\begin{gathered}
\mathrm{TT}=\mathrm{CT}+\mathrm{VT}=\left(\mathrm{T}_{\mathrm{d} 850}-\mathrm{T}_{500}\right)+\left(\mathrm{T}_{\mathrm{d} 850}-\mathrm{T}_{500}\right) \\
\mathrm{TT}=\mathrm{T}_{850}+\mathrm{T}_{\mathrm{d} 850}-2^{*} \mathrm{~T}_{500}
\end{gathered}
$$

Este índice tem como maior limitação a dependência da umidade em $850 \mathrm{hPa}$, assim como o índice K. O índice TT exige calor e umidade em $850 \mathrm{hPa}$ e frio em $500 \mathrm{hPa}$ e não restringe a umidade em $700 \mathrm{hPa}$. Se houver uma camada seca em $700 \mathrm{hPa}$ isso não afeta o valor de TT (SILVA DIAS, 2000).

Os valores de $\mathrm{K}>20$ indicam que existem possibilidades de chuvas isoladas, e à medida que esses valores crescem, aumentam as tempestades associadas, de acordo com George (1960). Já para o TT, o limiar tabelado (MILLER, 1972) para um potencial fraco de atividades convectivas é $\mathrm{TT}<44$. Ambos os índices e limiares foram desenvolvidos para latitudes médias do hemisfério norte. Assim, torna-se necessário futuramente estabelecer novos limiares para América do Sul. Os valores climatológicos (médios) determinados no presente estudo servem como um indicativo de atmosfera média, para que valores e previsões de índices muito acima da média sirvam de alerta para o previsor de tempo, porém estudos mais detalhados para cada área são necessários para estabelecer novos limiares por regiões.

\subsection{Teste de Hipótese}

A análise estatística de tendência foi realizada para identificar as regiões onde os valores dos índices de instabilidade têm aumentado ou diminuído ao longo do período analisado. Para o cálculo das tendências foi utilizada a técnica de regressão linear utilizando o método dos mínimos quadrados, e plotado nos mapas o coeficiente angular da reta. Foi utilizado o teste $t$ de significância para o coeficiente $r$ a fim de verificar se as tendências eram significativas com $90 \%$ de confiança. Esse método foi calculado a partir da equação (STEVENSON, 1981):

$$
t=\frac{r}{\sqrt{\frac{1-r^{2}}{n-2}}}
$$

Onde $r$ é o coeficiente correlação de Pearson, $n$ é o número de observações (com $n$-2 graus de liberdade) e $t$ é o valor do teste $t$. Para testar a significância dos valores, foi observado se o valor alegado está incluído ou não intervalo de confiança. Caso esse valor estivesse no intervalo, aceitaria-se a hipótese nula (H0) que consiste em não haver tendência nos índices, caso contrário, rejeitaria-se a hipótese nula e aceitaria-se a hipótese alternativa (H1), que indica a ocorrência de tendência nos dados.

\section{Resultados e Discussões}

Os índices $\mathrm{K}$ e TT bem como a tendência destes foram calculados para toda a América do Sul, e a análise climatológica foi realizada para os quatro horários sinóticos, sazonalmente.

As variações horárias entre os valores dos índices de instabilidade são explicadas pela variação diurna da temperatura, porém devido à semelhança no comportamento dos índices na climatologia dos horários analisados, a representação gráfica dos resultados é mostrada para o horário das $00 Z$, para que também pudessem ser comparados a outros estudos encontrados na literatura, como o de Escobar e Andrade (2010).

Os menores valores de índices de instabilidade indicam que o perfil atmosférico encontra-se mais estável não apresentando possibilidade de chuvas.

Devido à atuação do regime monçônico em parte do continente, é possível constatar que os maiores valores de ambos os índices encontram-se no verão, considerada a estação chuvosa.

A maior umidade está geralmente presente na atmosfera em baixos níveis e as maiores temperaturas registradas nesta estação fazem com que o valor dos 


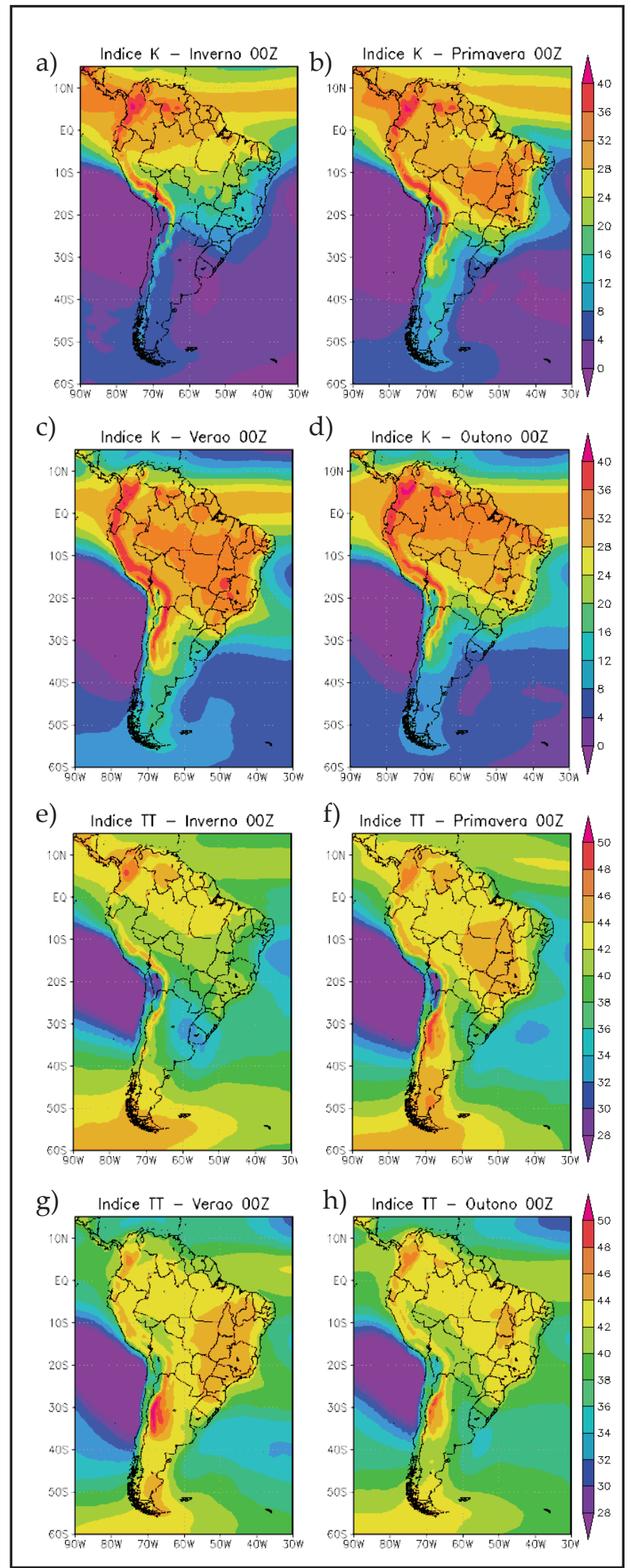

Figura 1 - Climatologia dos índices de instabilidade K (painel superior) e TT (painel inferior) para a América do Sul de 1979 a 2012 às 00Z: no inverno austral (a-e), primavera (b-f), verão (c-g) e outono (d-h) índices aumente (Figura 1.b; 1.c; 1.f; 1.g) em relação aos menores valores encontrados no inverno (Figura 1.a; 1.e), estação mais seca e com temperaturas mais brandas.

Na parte norte do continente é possível notar que os maiores valores dos índices $\mathrm{K}$ estão no outono (Figura 1.d) e do TT no inverno (Figura 1.e) devido a esta parte do continente se localizar no hemisfério norte, apresentando comportamento inverso ao do restante do continente.

A Figura 2 mostra os resultados da tendência linear para os índices Ke TT às 00Z. Para a análise de tendência, as linhas azuis correspondem à tendência negativa e as vermelhas referem-se à tendência positiva dos índices. Na figura em análise é possível notar que as estações do ano com maiores tendências positivas no valor do índice K são verão e primavera, onde obtêm-se valores significativos na região Amazônica e parte da região Centro Oeste do Brasil. Há tendência negativa nos valores principalmente no outono na Argentina (Figura 2.d; 2.h).

A tendência de aumento encontrada em ambos os índices pode estar relacionada ao crescente desmatamento na região Amazônica, e também com aumentos de temperatura previstos pelos cenários do IPCC (2013), nos quais, todas as projeções indicam aumento de temperatura para esta região.

Segundo Marengo (2006), os modelos mostram que na hipótese de desmatamento total da Amazônia, a temperatura e a evaporação tendem a aumentar, enquanto que a precipitação tende a diminuir. Estas mudanças podem ser resultado de interações entre o clima, hidrologia, vegetação e o gerenciamento dos recursos água e terra. Simulações realizadas por Oliveira (2008) indicam que a alteração da cobertura vegetal (causada pelo desmatamento) gerou mudanças na estrutura dinâmica da atmosfera em baixos níveis (na Camada Limite Planetária - CLP) e, consequentemente, na convergência de umidade. $\mathrm{O}$ aumento do aquecimento na CLP, causado pelo aumento no fluxo de calor sensível e pela diminuição da evapotranspiração sobre as áreas desflorestadas, induzem a uma convergência termicamente forçada, que pode resultar em um aumento da precipitação na região central (Estado do Amazonas) e oeste da Amazônia, onde também há tendência de aumento dos índices.

Como estes índices analisados dependem de temperatura, o aumento mais significativo dessa variável em camadas mais baixas da atmosfera são capazes de aumentar os valores obtidos pela climatologia. A tendência de aumento nos valores dos índices de instabilidade está presente principalmente nas áreas e estações do ano nas quais há maior atividade convectiva. Isso pode ser um indicativo de aumento na quantidade de sistemas convectivos ou, ainda, um aumento na intensidade da atividade convectiva o que deve ser analisado observando os padrões e cenários associados às mudanças climáticas. 

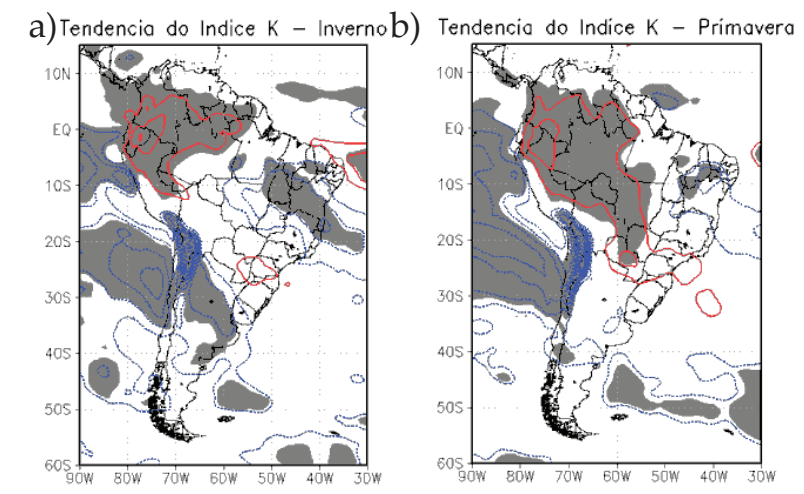

C) Tendencia do Indice $\mathrm{K}-$ Verao
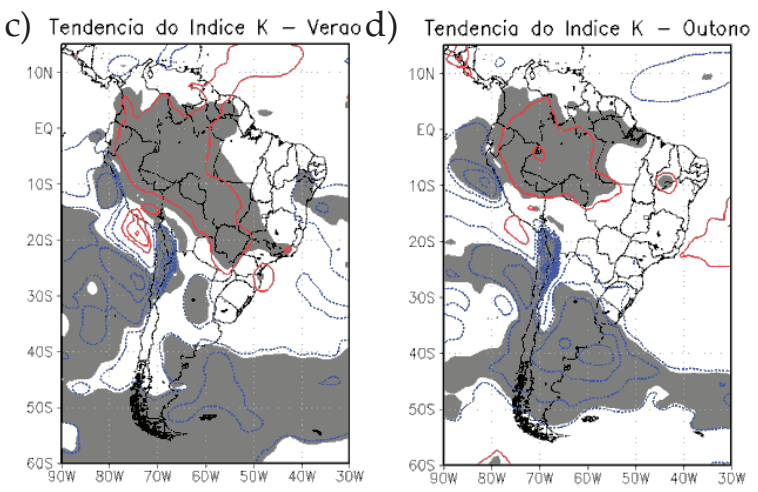

e) Tendencia do Indice TT - Inverno
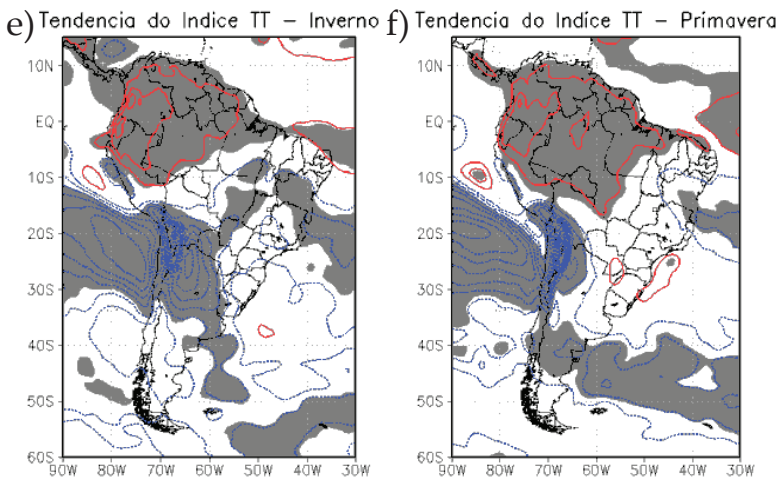

g) Tendencia do Indice $\Pi-$ Verao

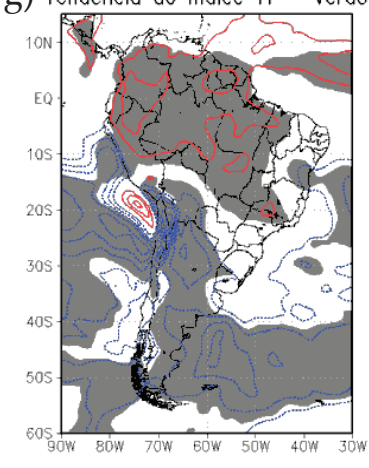

h) Tendencio do Indice TT - Outono

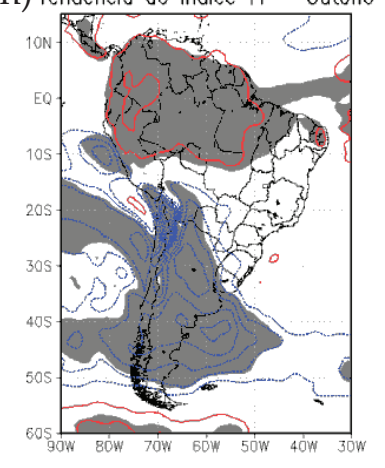

Figura 2: Tendência dos índices de instabilidade $\mathrm{K}$ (painel superior) e TT (painel inferior) para a América do Sul de 1979 a 2012 às 00Z: no inverno austral (a-e), primavera (b-f), verão (c-g) e outono (dh). As linhas vermelhas (azuis) indicam tendência de aumento (decréscimo) dos índice. Em cinza as áreas onde o coeficiente angular da reta é significativo ao nível de $90 \%$ de confiança.

\section{Conclusões}

Nas variações sazonais dos índices é possível observar que embora na região amazônica não há grandes variações sazonais, no verão e primavera a evaporação na região amazônica afeta a estrutura termodinâmica da atmosfera principalmente no sudeste do Brasil devido às zonas de convergências de umidade atuantes nessas estações. Por isso são encontrados maiores variações nos índices de instabilidade na região sudeste. $\mathrm{O}$ inverno apresenta menores valores dos índices para a maior parte do Brasil o que pode ser explicado pelo regime pluviométrico anual em parte do continente.

O cálculo da tendência linear pode auxiliar a previsão do tempo estabelecendo novos limiares locais mais elevados ou baixos para indicar possibilidade de tempestades. Há valores significativos de tendência de aumento de ambos os índices na região amazônica e tendência de diminuição nos valores dos índices na região da Argentina. Há evidências de que o aumento das atividades humanas (como mudanças no uso da terra e desmatamento) modificam as características termodinâmicas da baixa atmosfera, e esta é uma das possíveis causas da tendência de aumento dos índices na Amazônia.

Diversas pesquisas mostram que não há unanimidade em relação à tendência de precipitação na América do Sul, e com base nisso, trabalhos futuros podem ser desenvolvidos a fim de provar a real causa da tendência, assim como, estabelecer novos limiares dos índices para regiões específicas.

\section{Agradecimentos}

A PRPPG - Pró-Reitoria de Pesquisa e Pós-Graduação - da UNIFEI e a FAPEMIG pelos recursos disponibilizados para a apresentação parcial deste trabalho no $\mathrm{V}$ SIC - Simpósio Internacional de Climatologia, com o título "Índice de instabilidade $\mathrm{K}$ na região sudeste do Brasil". A Pós-Graduação em Meteorologia do INPE pelos recursos disponibilizados para a apresentação do presente trabalho. A primeira autora agradece a CAPES pela bolsa de mestrado.

\section{Referências}

BENETI CAA, SILVA DIAS MA. Análise da performance de índices de instabilidade como previsores de tempestades na região de São Paulo. In: Anais do IV Congresso Brasileiro de Meteorologia; Brasília-DF, 1986.

ESCOB AR GCJ, Andrade KM. Climatologia dos índices de instabilidade Ke Total Totals (TT) para o Sul e Sudeste do Brasil. In: Anais do XVI Congresso Brasileiro de Meteorologia, Belém-PA. 2010. 
FRAGOSO M. A utilização de índices de estabilidade no estudo de situações convectivas: o exemplo dos temporais de Outono de 1997, em Lisboa. Finisterra: Revista Portuguesa de Geografia, 1998.

George JJ. Weather Forecasting for Aeronautics. New York, Academic Press. 1960. 673 pp.

INTERGOVERNMENTAL PANEL ON CLIMATE CHANGE. Climate Change 2013 (IPCC). The physical science basis: Working group I contribution to the fifth assessment report of the Intergovernmental Panel on Climate Change. Cambridge University Press, 2014.

MARENGO J. Caracterização do clima no Século XX e Cenários Climáticos no Brasil e na América do Sul para o Século XXI derivados dos Modelos Globais de Clima do IPCC. 2006.

MILLER RC. Notes on analysis and severe-storm forecasting procedures of the Air Force Global Weather Central. Air Weather Service, United States Air Force. 1972. 190 pp.

NASCIMENTO EL. Previsão de tempestades severas utilizando-se parâmetros convectivos e modelos de mesoescala: Uma estratégia operacional adotável no Brasil? Revista Brasileira de Meteorologia. 2005. (20):121140.

OLIVEIRA GS. Consequências climáticas da substituição gradual da Floresta Tropical Amazônica por pastagem degradada ou por plantação de soja: um estudo de modelagem- [thesis]. São José dos Campos: INPE; 2008. 417p.

PINHEIRO HR, ESCOBAR GCJ, Andrade KM. Aplicação de uma ferramenta objetiva para previsão de tempo severo em ambiente operacional. Revista Brasileira de Meteorologia, 2013. (29) 2.

SILVA DIAS MAF. Índices de instabilidade para a previsão de chuva e tempestade severas. [Internet] Universidade de São Paulo, São Paulo; 2000. [cited 2015 Jun 10]. Available from: www.master.iag.usp.br/static/ downloads/apostilas/indices.pdf

Stevenson W. Estatística aplicada à administração. Harper \& Row do Brasil, São Paulo, 1981.

VIANELLO RL, ALVES AR. Meteorologia Básica e Aplicações. Viçosa, MG., UFV - Imprensa Universitária, 1991.(449) 